\title{
Non-Biological Membranes as Drug Delivery Systems
}

\author{
Shankar Swaminathan and Monica M. Jablonski* \\ Department of Ophthalmology, Hamilton Eye Institute, The University of Tennessee Health Science Center, Memphis, TN, USA
}

Drug delivery, in its simplest, most common form, is comprised of fast-acting chemical compounds that are dispensed either topically or systemically. In actual sense, polymeric drug delivery systems can be considered as non-biological membranes encapsulating drug and controlling the release as the drug partitions across the membrane to reach the target site. They can be engineered to deliver the right amount of the drug in the right place. The focus of this editorial will be on the eye and issues concerning two highly prevalent eye diseases - age related macular degeneration (AMD) and glaucoma. Theoretically, an ideal drug delivery system for eye diseases is the one which is given topically, once in six months, releases the active at the site in a controlled manner, without any side effects or vision distortions. This elusive "Ideal Drug Delivery System" leaves a lot to be desired. Blindness and irreversible sight impairment due to eye diseases cost an estimated $\$ 50$ billion each year in the United States and, thus, improving on the treatments available today could substantially reduce the financial burden on society.

While mainly posterior eye based delivery methodologies are used in case of $\mathrm{AMD}$, anterior drug delivery technologies rule the roost in case of glaucoma treatment options. Delivery of drugs to the posterior eye is challenging, owing to anatomical and physiological constrains of the eye. Currently, the intravitreal route is widely used to deliver therapeutic entities to the retina. It involves direct injection of the formulation, in the form of solution, particles, suspension, depot or implants, into the posterior segment through the pars plana. Intravitreal injection provides increased drug concentrations at the neural retina and minimizes systemic side effects. The focus of the research in this domain has been the development of methods that will increase the efficacy of spatiotemporal drug application, resulting in more successful therapy for patients. Intravitreal injections display first-order kinetics (drug efficacy can diminish as the drug concentration falls below the targeted range), injections have short half-life (few hours), and should be administered repeatedly, side effects which include pain caused by repeated injections, discomfort, increased IOP, intraocular bleeding, increased chances for infection, floaters, tear formation, cataracts, transient blurry vision, and the possibility of retinal detachment; the major complication for intravitreal injection is endophthalmitis. All these drawbacks lead to poor acceptance by patients. Controlled delivery systems, such as biodegradable and non-biodegradable implants, liposomes and nanoparticles, have been developed to overcome such adverse effects with some success.

While no FDA approved macular degeneration treatments options lead to a complete restoration of vision lost to the eye disease, a few possibilities such as Lucentis, Eyelea and some other investigational drug approaches may preserve or even improve the remaining vision. Other than the implantable telescope, following are the FDA approved drug/biological regimens: Lucentis, Macugen, Eyelea, Visudyne, and AERDS nutritional formula. Other investigational approaches are Avastin, iSONEP, Forsight VISION4 implant, MC-1101 with VersiDoser ${ }^{\mathrm{TM}}$ delivering a spray plume, Evizon, Zybrestat, Fenretinide, NT-501, radiation treatment, artificial retinas, gene therapy and stem cells. Most of the approaches, both FDA and non-FDA are however for the wet form of AMD. There is a huge technological gap and an untapped market potential for development of drug delivery systems for dry from of AMD. Even the existing systems for wet AMD suffer from drawbacks of repeated injections; cost, stability of formulations etc. thereby leading to patient noncompliance.

While being the least invasive method of drug application, topically applied drugs are hindered by many components of the anterior eye, including the corneal epithelium, corneal endothelium, conjunctiva and sclera. Also, the longer diffusion distance to the posterior eye and the acellular nature of the vitreous negatively impact the pharmacokinetics and distribution of the topical drugs. Simple physiologic processes such as tear production, blinking, drug metabolism and drug binding also impact topical applications, hindering the access of topical drugs to the target locations. All of these factors and limitations lead to increased dosing and a higher frequency of drug application in order to attain therapeutic concentrations, making the use of topical drugs relatively inefficient for patients and leading to decreased patient noncompliance due to repeated dosing requirement and inability to instill the drops due to shaky hands in case of older patients. More than fifty percent of the glaucoma patients thus discontinue medications due to noncompliance. The sustained local drug level should be therapeutically beneficial for enhanced efficiency, and reduced dosage may be desired for less systemic toxicity. In situ gelling systems with polymers like poloxamers, bioadhesive polymers like hyaluronic acid, chitosan, polycarbophils, gums etc. have been used as retention enhancers, permeation enhancers like cyclodextrins and surfactants, $\mathrm{pH}$ and temperature sensitive hydrogels, cationic liposomes, dendrimers, niosomes, microemulsions and polymer based nanoparticulate systems, viscosity enhancers, prodrugs etc. have been used. A hybrid approach utilizing nanoparticles/microparticles in a drop/gel formulation offers an added advantage of enhanced bioavailability due to effective permeation and targeting to the site. Reported examples include liposomes and nanoparticles in droppable gels and liposomes and nanoparticles coated with bioadhesive polymers.

Thus, the goal of pharmacotherapeutics is to treat a disease in a consistent and predictable fashion. A significant challenge to the formulator is to circumvent the protective barriers of the eye without causing permanent tissue damage. Need of the hour is to synthesize new class of polymers/ utilize the existing ones to formulate biodegradable systems, using newer economic chemistries, easily

*Corresponding author: Dr. Monica M Jablonski, Department of Ophthalmology, Hamilton Eye Institute, The University of Tennessee Health Science Center, 930 Madison Avenue, Suite 731, Memphis, TN 38163, USA, Tel: (901) 448-7572; Fax: (901) 448-5028; E-mail: mjablonski@uthsc.edu

Received July 18, 2012; Accepted July 19, 2012; Published July 23, 2012

Citation: Swaminathan S, Jablonski MM (2012) Non-Biological Membranes as Drug Delivery Systems. J Memb Sci Technol 2:e108. doi:10.4172/21559589.1000e108

Copyright: (c) 2012 Swaminathan S, et al. This is an open-access article distributed under the terms of the Creative Commons Attribution License, which permits unrestricted use, distribution, and reproduction in any medium, provided the original author and source are credited. 
scalable, and reproducible. The developed system must also give a reasonable loading of drug irrespective of the molecular size, charge, and Biopharmaceutical Classification System class thereby providing affordable treatment options. It must also offer reproducible spherical sizes, tunable zeta potentials and predictable physiological behavior at a molecular and cellular level. The polymeric systems must also comply with the ISO standards of safety and biocompatibility. Comprehensive lab studies and clinical studies including efficacy in animal models should be a part of the drug development protocol.

In future, much of the emphasis will be given to achieve noninvasive sustained drug release for eye disorders in both segments. A clear understanding of the complexities associated with tissues in normal and pathological conditions, physiological barriers, and multicompartmental pharmacokinetics would greatly hasten further development in the field. The ocular bioavailability must be increased from less than $1 \%$ to $15-20 \%$ of the administered dose. Most of the currently marketed ocular drugs were initially developed for nonocular applications, hence their low or nonspecificity. So, there is a need to develop new drug candidates primarily intended for ocular use.

An ideal system should be able to achieve an effective drug concentration at the target tissue for an extended period of time, while minimizing systemic exposure. In addition, the system should be both comfortable and easy to use. Patient acceptance will continue to be emphasized in the design of future ophthalmic drug delivery systems. A reasonable strategy to circumvent the drawbacks of individual technologies is to combine technologies. Tailoring of the delivery systems based on the surface charge, polymer coating thickness and particle sizes would also lead to a plethora of delivery options. Time is right for an explosion of new drug formulations that include nonbiological membranes as barriers that must be crossed to deliver drug into various compartment of the body. It is fascinating to note the impact that the membrane characteristics have on the drug eluting properties. Advanced membrane characterization techniques can directly be used for understanding the drug partitioning behavior and eventual release kinetics. It could be interesting to study the drug release characteristics with an emphasis on membrane properties like plasticity, elastic modulus, tensile strength etc., Herein, we used the eye as an example but the same principles can be applied to other organs systems. Science and medicine can and must merge to deliver more effective therapies to enhance our lives.

\section{Acknowledgements}

Supported by NEI Core Grant P30EY013080; The University of Tennessee Research Foundation and an Unrestricted Grant from Research to Prevent Blindness, New York, NY. 